\title{
Electroweak Interaction Model with an Undegenerate Double Symmetry
}

\author{
Leonid M. SLAD
}

D.V. Skobeltsyn Institute of Nuclear Physics, Moscow State University, Moscow, 119899 Russia

E-mail: slad@theory.sinp.msu.ru

Received December 12, 2005, in final form March 31, 2006; Published online April 20, 2006

Original article is available at http://www.emis.de/journals/SIGMA/2006/Paper045/

\begin{abstract}
The initial $P$-invariance of the electroweak interaction Lagrangian together with the low-energy results of the Weinberg-Salam model is provided by a local secondary symmetry. Among the transformation parameters of this symmetry there are both scalars, and pseudo-scalars with respect to the orthochronous Lorentz group. Such symmetry does admissible existence of a light (massless) axial gauge boson and its possible nonuniversal interaction with the leptons of various types.
\end{abstract}

Key words: double symmetry; electroweak interactions; light axial gauge boson

2000 Mathematics Subject Classification: 81T10; 81R05; 81V10

\section{Introduction}

The basic point of this report is a logic precondition for possible existence of a light (massless) axial gauge boson which may interact with the electronic neutrino. This precondition is based on a symmetry approach, which has been formulated rather recently, though separate examples of its realization are known for a long time. Prior to passing to the basic theme of the report, we consider it necessary to give some characteristics of the double symmetry concept used by us, to list the existing undegenerate double symmetries in field theories, and to formulate the principal positions of the initially $P$-invariant model of electroweak interactions.

\section{Some characteristics of the double symmetry}

The concepts of the secondary and double symmetry is proposed by us [1] as some generalization of already existing approaches to the field theories construction.

The double symmetry group $\mathcal{G}_{T}$ is always the semidirect product $\mathcal{G}_{T}=H_{T} \circ G$ of the subgroup $G$, which is the global primary symmetry group, and the invariant subgroup $H_{T}$, which is the global or local secondary symmetry group.

The secondary symmetry group $H_{T}$ is generated by transformations, whose parameters $\theta=$ $\left\{\theta_{a}\right\}$ are vectors of the space of a set beforehand representation $T$ of the primary symmetry group $G$. Let us emphasize that in the general group theory, the situation when the parameters of one group transform as a nontrivial representation of another one is not discussed at all.

We realize both a representation $S$ of the primary symmetry group $G$, and the secondary symmetry transformations in the form

$$
\Psi^{\prime}(x)=\exp \left(-i D^{a} \theta_{a}\right) \Psi(x)
$$

in the same space of field vectors $\Psi(x)$. Generally the secondary symmetry transformations (1) connect among themselves both the vectors of the same irreducible representation, and the vectors of various irreducible representations belonging to the representation $S$ of group $G$. 
The secondary symmetry transformations do not violate the primary symmetry. It means that the operators $D^{a}$ in equation (1) should be the $T$-operators of the group $G$, i.e.

$$
D^{a}=S^{-1}(g) D^{b} S(g)[T(g)]_{b}{ }^{a} .
$$

If the secondary symmetry is produced by the adjoint representation of the group $G$ and the operators $D^{a}$ in equation (1) coincide with the group generators, then obviously the group $H_{T}$ is locally isomorphic to the group $G$. In such a case we say that the double symmetry is degenerate.

\section{Existing undegenerate double symmetries in field theories}

A. The $\sigma$-model symmetry. The $\sigma$-model symmetry of Gell-Mann and Levy [2] is nothing but an undegenerate double symmetry. Transformations in the $\sigma$-model connect the pseudoscalar $\pi$-meson and the scalar $\sigma$-meson. Their infinitesimal form is

$$
\begin{aligned}
\boldsymbol{\pi}^{\prime} & =\boldsymbol{\pi}+i \boldsymbol{\theta} \sigma \\
\sigma^{\prime} & =\sigma-i \boldsymbol{\theta} \boldsymbol{\pi} .
\end{aligned}
$$

Gell-Mann and Levy obviously note that in transformations (2) and (3) the parameter $\boldsymbol{\theta}$ is a pseudoscalar with respect to the orthochronous Lorentz group $L^{\uparrow}$. Due to this, these transformations do not violate the spatial reflection symmetry $\mathrm{P}$.

In the $\sigma$-model, the primary symmetry group $G$ is $S U(2) \otimes L^{\uparrow}$. The secondary symmetry is produced by the representation $T=$ (isotriplet, pseudoscalar) of $G$. Its group is $H_{T}=$ $S U(2)_{L} \otimes S U(2)_{R}$. The parameters of one of the groups $S U(2)$ are given by the sum of the space scalar and pseudoscalar, and the parameters of the other group are given by their difference. It is necessary specially to note that, unlike Gell-Mann and Levy, nobody except us [1] said anything about the $P$-properties of parameters of the chiral symmetry group $S U(2)_{L} \otimes S U(2)_{R}$.

B. Supersymmetry. Supersymmetry, both in the $x$-space and the superspace, can be considered as a secondary symmetry produced by the bispinor representation (by a direct sum of two nonequivalent irreducible spinor representations) of the proper Lorentz group $L_{+}^{\uparrow}$, which plays the role of the primary symmetry group. The set of generating elements for supersymmetry consist of transformations of the form (1), in which the parameters $\theta=\left\{\theta_{a}\right\}$ belong to the bispinor representation space of the group $L_{+}^{\uparrow}$. Therefore, supersymmetry transformations connect bosonic and fermionic fields. This connection does not break the statistics of the field states, iff the parameters $\theta_{a}$ are anticommutating elements of the Grassmann algebra.

C. The Poincarè group $\mathcal{P}$ as a double symmetry group. It is well-known that the Poincarè group is the semidirect product $\mathcal{P}=T_{4} \circ L^{\uparrow}$ of the subgroup of orthochronous Lorentz transformations $L^{\uparrow}$ and the invariant subgroup of four-dimensional translations $T_{4}$. Therefore, the translation group can be considered as a secondary symmetry group produced by the polar four-vector representation of the orthochronous Lorentz group, and thus, $L^{\uparrow}$ is the primary symmetry group. The corresponding secondary symmetry (translation) transformations have the form

$$
\Psi^{\prime}(x)=\exp \left(-i D^{\mu} \theta_{\mu}\right) \Psi(x),
$$

where the parameters $\theta_{\mu}$ are four-vector translations, and $D^{\mu}$ are the differential operators, namely $D^{\mu}=i \partial^{\mu}$.

D. An infinite-component field theory with a double symmetry. We have received a number of physically interesting results in the theory of ISFIR-class fields, which transform 
as proper Lorentz group representations decomposable into an infinite direct sum of finitedimensional irreducible representations $[3,4,5,6]$.

The infinite number of arbitrary parameters in the relativistically invariant Lagrangians of the free ISFIR-class fields was the serious reason for that until recently, there were no research of such field theory. This arbitrariness is eliminated by the requirement for the theory to be invariant also under secondary symmetry transformations of the form (4), in which the parameters $\theta_{\mu}$ are the components of the polar or axial 4-vectors of the orthochronous Lorentz group, and the operators $D^{\mu}$ have the matrix realization.

It is proved [6] that there are such theories of the ISFIR-class fields with spontaneously broken secondary symmetry, whose mass spectra are quite satisfactory from the standpoint of particle physics. This result is especially important, because in all previous attempts aimed at a consistent relativistic description of particles with an infinite number of degrees of freedom, the mass spectra were physically unacceptable, as they had an accumulation point at zero. Let us note that such attempts were undertaken in particular by the well-known physicists V.L. Ginzburg, I.E. Tamm, H. Yukawa, Yu.M. Shirokov, and M.A. Markov (see the review paper [7]) and mathematicians I.M. Gelfand and A.M. Yaglom (see the monograph [8]).

\section{Initially $\boldsymbol{P}$-invariant electroweak model as a logical correction of the standard left-right symmetric model}

The Weinberg-Salam electroweak model with the gauge group $S U(2)_{L} \otimes U(1)$ is initially asymmetric with respect to the left-handed and right-handed spinors and, therefore, noninvariant with respect to the spatial reflection $P$.

The standard left-right symmetric model of electroweak interactions $[9,10,11,12]$ eliminating the mentioned asymmetry reproduced all results of the Weinberg-Salam model in the region of existing energies. Possible manifestations at high energies of the additional heavy bosons, denoted as $W_{R}$ and $Z_{L R}$, are a subject of regular experimental studies (see, for example, [13]). The parameters of the gauge group $S U(2)_{L} \otimes S U(2)_{R} \otimes U(1)$ of $[9,10,11,12]$ are scalars with respect to orthochronous Lorentz group $L^{\uparrow}$. Such group transformations violate the $P$ symmetry. For example, they transform a $P$-even fermionic state into a state with uncertain $P$-parity. Thus, the left-right symmetry of $[9,10,11,12]$ does not entail the $P$-symmetry.

The concept of the left or right essence is logically strict only concerning the irreducible spinor representations of the proper Lorentz group $L_{+}^{\uparrow}$ and the currents constructed of these spinors. It does not bear in itself any sense concerning the integer spin fields and, hence, concerning the gauge and Higgs fields. The mathematically exact concept, which bears a direct relation to all elements of electroweak interactions, is the $P$-transformation of any field, any current, etc. The $P$-transformation converts, in particular, the left-handed spinor into the right-handed one and vice versa. The $P$-symmetry entails the left-right symmetry.

If we wish to have the initially $P$-invariant model of electroweak interactions, it is necessary to construct it in the spirit of Gell-Mann and Levy [2]. Such construction has been realized in the paper [1], where initial $P$-invariance and its observed violation has been ensured by the local secondary symmetry produced by the representation $T=($ isotriplet, scalar $) \oplus$ (isotriplet, pseudoscalar $) \oplus$ (isosinglet, scalar $) \equiv(1, s) \oplus(1, p) \oplus(0, s)$ of the primary symmetry group $G=S U(2) \otimes L^{\uparrow}$. The corresponding parameters of the secondary symmetry transformations and the gauge fields are denoted as $\boldsymbol{\theta}^{1 s}=\left\{\theta_{j}^{1 s}\right\}, \boldsymbol{\theta}^{1 p}=\left\{\theta_{j}^{1 p}\right\}, \theta^{0 s} ; \boldsymbol{B}_{\mu}^{1 V}=\left\{B_{j \mu}^{1 V}\right\}, \boldsymbol{B}_{\mu}^{1 A}=\left\{B_{j \mu}^{1 A}\right\}$, $B_{\mu}^{0 V}(j=1,2,3$; the indices $V$ and $A$ mean the polar and axial 4-vectors, respectively).

As the gauge groups of both the standard left-right symmetric model of electroweak interactions $[9,10,11,12]$, and the primary $P$-invariant model [1] are locally isomorphic, the Lagrangian structure for these models and, hence, all their numerical results are identical. But in the con- 
ceptual plan, the initially $P$-invariant model gives several remarkable consequences which are absent in the left-right symmetric one.

First, the Higgs field $\Phi$ should have both scalar, and pseudoscalar components, namely, the isodoublets $\phi^{\frac{1}{2} s}$ and $\phi^{\frac{1}{2} p}$. If the neutral components of both of them take some nonzero vacuum

expectation values $\left\langle\phi_{-1 / 2}^{\frac{1}{2} s}\right\rangle=v_{s}$ and $\left\langle\phi_{-1 / 2}^{\frac{1}{2} p}\right\rangle=v_{p}$ with $\arg \left(v_{p} / v_{s}\right) \neq \pm \pi / 2$, then the masses of the two $W$-bosons, which are connected with the left and right charged currents, are different, i.e. the $P$-symmetry is violated. Hence the nature of $P$-symmetry violation is in that the physical vacuum does not possess definite $P$-parity. Let us note that the relation

$$
\left|v_{s}-v_{p}\right| \ll\left|v_{s}+v_{p}\right|
$$

is a sufficient condition for reproducing all results of the Weinberg-Salam model, which are obtained for already completed experiments.

Second, the fields of all intermediate bosons constitute a superposition of polar and axial 4 -vectors, and these vectors have equal weight in the fields of $W$-bosons. In other words, the $P$-properties of the weak currents and the $W$ - and $Z$-bosons connected with the corresponding currents are similar.

The formulas, confirming these two statements, will be presented in the following section.

\section{$5 \quad$ Initially $\boldsymbol{P}$-invariant electroweak model with the light (massless) axial gauge boson}

Look at the parameters of the local group of the secondary symmetry, which are listed in the previous section. The isotriplet parameters are both scalar, and pseudoscalar with respect to the group $L^{\uparrow}$. But, among the isosinglet parameters, we find only a scalar and we do not find a pseudoscalar. Such inequality does not look sufficiently natural. We hope to analyse in the future the physical consequences of its elimination, laying here the grounds for such analysis.

So, we shall enlarge the list of parameters of the secondary symmetry group, leading to the initially $P$-invariant model of electroweak interactions, by one more parameter $\theta^{0 p}$ which transforms as the representation (isosinglet, pseudoscalar) of the primary symmetry group $G=$ $S U(2) \otimes L^{\uparrow}$. The corresponding field will be denoted as $B_{\mu}^{0 A}$.

In the fermionic sector we restrict ourselves to isodoublet $\psi^{T}=\left(\nu_{e}, e\right)$ consisting of the fields of electronic neutrino $\nu_{e}$ and electron $e$. Its transformations of the secondary symmetry under considaration can be written in the form

$$
\psi^{\prime}=\exp \left(-\frac{i}{2} \boldsymbol{\tau} \boldsymbol{\theta}^{1 s}-\frac{i}{2} \gamma^{5} \boldsymbol{\tau} \boldsymbol{\theta}^{1 p}+\frac{i}{2} \theta^{0 s}+\frac{i c_{1}}{2} \gamma^{5} \theta^{0 p}\right) \psi,
$$

where $\tau$ are Pauli matrices and $c_{1}=0$ or $2 \sqrt{2}$.

The global secondary symmetry transformations of the fields $\boldsymbol{B}_{\mu}^{1 V}$ and $\boldsymbol{B}_{\mu}^{1 A}$ are

$$
\left(\begin{array}{l}
\boldsymbol{B}_{\mu}^{1 V} \\
\boldsymbol{B}_{\mu}^{1 A}
\end{array}\right)^{\prime}=\exp \left[-i\left(\begin{array}{cc}
\boldsymbol{t} & 0 \\
0 & \boldsymbol{t}
\end{array}\right) \boldsymbol{\theta}^{1 s}-i\left(\begin{array}{ll}
0 & \boldsymbol{t} \\
\boldsymbol{t} & 0
\end{array}\right) \boldsymbol{\theta}^{1 p}\right]\left(\begin{array}{l}
\boldsymbol{B}_{\mu}^{1 V} \\
\boldsymbol{B}_{\mu}^{1 A}
\end{array}\right),
$$

where $\boldsymbol{t}=\left\{t_{j}\right\}(j=1,2,3)$ are the generators of adjoint representation of the group $S U(2)$.

The doubly symmetric interaction Lagrangian of the gauge field and leptonic field $\psi$ is fixed in the form

$$
\mathcal{L}_{\mathrm{int}}=-\frac{1}{2 \sqrt{2}} \bar{\psi}\left(g \gamma^{\mu} \boldsymbol{\tau} \boldsymbol{B}_{\mu}^{1 V}+g \gamma^{\mu} \gamma^{5} \boldsymbol{\tau} \boldsymbol{B}_{\mu}^{1 A}-g_{0} \gamma^{\mu} B_{\mu}^{0 V}-c_{1} g_{A} \gamma^{\mu} \gamma^{5} B_{\mu}^{0 A}\right) \psi,
$$

where the coupling constants $g, g_{0}$ and $g_{A}$ are positive. 
We write down the transformations of the Higgs field $\Phi$ and mass terms of the Lagrangian for the gauge fields

$$
\begin{aligned}
& \left(\begin{array}{c}
\phi^{\frac{1}{2} s} \\
\phi^{\frac{1}{2} p}
\end{array}\right)^{\prime}=\exp \left[-\frac{i}{2}\left(\begin{array}{cc}
\boldsymbol{\tau} & 0 \\
0 & \boldsymbol{\tau}
\end{array}\right) \boldsymbol{\theta}^{1 s}-\frac{i}{2}\left(\begin{array}{cc}
0 & \boldsymbol{\tau} \\
\boldsymbol{\tau} & 0
\end{array}\right) \boldsymbol{\theta}^{1 p}-\frac{i}{2} \theta^{0 s}-\frac{i c_{2}}{2}\left(\begin{array}{ll}
0 & 1 \\
1 & 0
\end{array}\right) \theta^{0 p}\right]\left(\begin{array}{c}
\phi^{\frac{1}{2} s} \\
\phi^{\frac{1}{2} p}
\end{array}\right), \\
& \mathcal{L}_{\text {mass }}=\left|\left(\mathcal{M}^{\mu}\right)^{\dagger} \mathcal{M}_{\mu}\right|,
\end{aligned}
$$

where

$$
\begin{aligned}
\mathcal{M}_{\mu}= & -\frac{1}{2 \sqrt{2}}\left[g\left(\begin{array}{ll}
\boldsymbol{\tau} & 0 \\
0 & \boldsymbol{\tau}
\end{array}\right) \boldsymbol{B}_{\mu}^{1 V}+g\left(\begin{array}{cc}
0 & \boldsymbol{\tau} \\
\boldsymbol{\tau} & 0
\end{array}\right) \boldsymbol{B}_{\mu}^{1 A}+g_{0} \boldsymbol{B}_{\mu}^{0 V}\right. \\
& \left.+c_{2} g_{A}\left(\begin{array}{ll}
0 & 1 \\
1 & 0
\end{array}\right) \boldsymbol{B}_{\mu}^{0 A}\right]\left(\begin{array}{c}
\left\langle\phi^{\frac{1}{2} s}\right\rangle \\
\left\langle\phi^{\frac{1}{2} p}\right\rangle
\end{array}\right)
\end{aligned}
$$

$c_{2}$ being an arbitrary constant.

As it was pointed out by Weinberg [14], an essential element in the analysis of any gauge theory is the reduction of the Lagrangian mass term to a diagonal form that means the elimination of field oscillations, i.e. the elimination of transitions of one fields into others. As a result of this procedure, there appear the orthonormalized physical fields with their masses and coupling constants. The physical fields represent linear sums (orthogonal rotation) of the initial gauge fields. In the Weinberg-Salam model, such rotation involves two neutral gauge fields and is characterized by one angle which is the Weinberg angle. In the model under consideration, an appropriate orthogonal rotation involves four neutral gauge fields and it is convenient not to introduce any angles.

As it follows from equations (8) and (9), only two neutral gauge fields of the four ones possess masses, and the other two fields are massless. One of the massless gauge fields $A_{\mu}$ is the electromagnetic field and is described by the polar four-vector

$$
A_{\mu}=\frac{1}{\sqrt{g^{2}+g_{0}^{2}}}\left(g_{0} B_{3 \mu}^{1 V}+g B_{\mu}^{0 V}\right) .
$$

The other massless gauge field $Y_{\mu}$ is described by the axial four-vector

$$
Y_{\mu}=\frac{1}{\sqrt{g^{2}+\left(c_{2} g_{A}\right)^{2}}}\left(c_{2} g_{A} B_{3 \mu}^{1 A}+g B_{\mu}^{0 A}\right) .
$$

The axial gauge field $Y_{\mu}$ can get some small mass due to another Higgs field (besides the field $\Phi$ ), or due to a mechanism we yet know nothing about.

Only such situation seems interesting to us when the axial gauge boson interacts with the electronic neutrino and there is an energy region in which this interaction can in principle be experimentally detected.

As to the interactions of the axial field $Y_{\mu}$ with other fields, there are two essentially different scenarios.

In the first variant the condition $c_{2} \neq 0$ is satisfied and consequently the field $B_{3 \mu}^{1 A}$ has a nonzero projection on the state $Y_{\mu}$. It means that the axial boson $Y$ interacts with one or two components of each fermionic isodoublet and with the massive gauge fields. The axial boson interacts with the electronic neutrino, if $c_{1} \neq c_{2}$.

In the second variant the constant $c_{2}$ is zero. Therefore, the axial gauge boson can interact only with fermions, and only with those ones, whose transformations of the type (5) really contain the parameter $\theta^{0 p}$. In particular, it interacts with the electronic isodoublet, if $c_{1} \neq 0$. So, in this variant of the electroweak model, the universality of all interactions of the charged or neutral leptons is not obligatory. 
The fields of comparatively light $W_{\mu}^{(1) \pm}, Z_{\mu}^{(1)}$ and heavy $W_{\mu}^{(2) \pm}, Z_{\mu}^{(2)}$ intermediate bosons and their masses are described by the following relations

$$
\begin{aligned}
& W_{\mu}^{(1) \pm}=\frac{1}{2}\left[\left(B_{1 \mu}^{1 V}-B_{1 \mu}^{1 A}\right) \mp i\left(B_{2 \mu}^{1 V}-B_{2 \mu}^{1 A}\right)\right], \\
& W_{\mu}^{(2) \pm}=\frac{1}{2}\left[\left(B_{1 \mu}^{1 V}+B_{1 \mu}^{1 A}\right) \mp i\left(B_{2 \mu}^{1 V}+B_{2 \mu}^{1 A}\right)\right], \\
& Z_{\mu}^{(1)}=\sqrt{\frac{g^{2}+g_{0}^{2}}{2 g^{2}+g_{0}^{2}}}\left[\frac{g}{g^{2}+g_{0}^{2}}\left(g B_{3 \mu}^{1 V}-g_{0} B_{\mu}^{0 V}\right)-B_{3 \mu}^{1 A}\right]+\mathcal{O}(x)+\mathcal{O}\left(g_{A}\right), \\
& Z_{\mu}^{(2)}=\frac{1}{g^{2}+g_{0}^{2}}\left(g B_{3 \mu}^{1 V}-g_{0} B_{\mu}^{0 V}+g B_{3 \mu}^{1 A}\right)+\mathcal{O}(x)+\mathcal{O}\left(g_{A}\right), \\
& m_{W^{(1)}}^{2}=\frac{g^{2}}{4}\left|v_{s}-v_{p}\right|^{2}, \\
& m_{W^{(2)}}^{2}=\frac{g^{2}}{4}\left|v_{s}+v_{p}\right|^{2}, \\
& m_{Z^{(1)}}^{2}=m_{W^{(1)}}^{2} \frac{2\left(g^{2}+g_{0}^{2}\right)}{2 g^{2}+g_{0}^{2}}\left[1-\frac{g_{0}^{2}}{g^{2}} x+\mathcal{O}\left(x^{2}\right)+\mathcal{O}\left(g_{A}^{2}\right)\right] \text {, } \\
& m_{Z^{(2)}}^{2}=m_{W^{(2)}}^{2} \frac{\left(2 g^{2}+g_{0}^{2}\right)}{2 g^{2}}\left[1+\frac{g_{0}^{2}}{g^{2}} x+\mathcal{O}\left(x^{2}\right)+\mathcal{O}\left(g_{A}^{2}\right)\right],
\end{aligned}
$$

where $x=g^{2} g_{0}^{2}\left(2 g^{2}+g_{0}^{2}\right)^{-2}\left|v_{s}-v_{p}\right|^{2}\left|v_{s}+v_{p}\right|^{-2}$.

The electroweak interaction Lagrangian is

$$
\begin{aligned}
& \mathcal{L}_{\text {int }}=e_{0} \bar{e} \gamma^{\mu} e A_{\mu}+\frac{g g_{A}}{2 \sqrt{2\left(g^{2}+\left(c_{2} g_{A}\right)^{2}\right)}}\left[\left(c_{1}-c_{2}\right) \bar{\nu}_{e} \gamma^{\mu} \gamma^{5} \nu_{e}+\left(c_{1}+c_{2}\right) \bar{e} \gamma^{\mu} \gamma^{5} e\right] Y_{\mu} \\
& -\frac{g}{2 \sqrt{2}}\left[\bar{\nu}_{e} \gamma^{\mu}\left(1-\gamma^{5}\right) e W_{\mu}^{(1)+}+\bar{\nu}_{e} \gamma^{\mu}\left(1+\gamma^{5}\right) e W_{\mu}^{(2)+}+\text { h.c. }\right] \\
& -\frac{g}{2} \sqrt{\frac{2\left(g^{2}+g_{0}^{2}\right)}{2 g^{2}+g_{0}^{2}}}\left[\bar{\nu}_{e}\left(\frac{1}{2}\left(1-\frac{2\left(g^{2}+g_{0}^{2}\right)}{g^{2}} x\right) \gamma^{\mu}-\frac{1}{2}(1+2 x) \gamma^{\mu} \gamma^{5}\right) \nu_{e}+\right. \\
& +\bar{e}\left(\left(-\frac{1}{2}+\frac{g_{0}^{2}}{g^{2}+g_{0}^{2}}\right)\left(1-\frac{2\left(g^{2}+g_{0}^{2}\right)}{g^{2}} x\right) \gamma^{\mu}+\frac{1}{2}(1+2 x) \gamma^{\mu} \gamma^{5}\right) e \\
& \left.+\mathcal{O}\left(x^{2}\right)+\mathcal{O}\left(g_{A}^{2}\right)\right] Z_{\mu}^{(1)} \\
& -\frac{g}{2} \sqrt{\frac{2 g^{2}}{2 g^{2}+g_{0}^{2}}}\left[\bar{\nu}_{e}\left(\left(\frac{1}{2}+\frac{g_{0}^{2}}{2 g^{2}}\right)(1+2 x) \gamma^{\mu}+\frac{1}{2}\left(1-\frac{2\left(g^{2}+g_{0}^{2}\right)}{g^{2}} x\right) \gamma^{\mu} \gamma^{5}\right) \nu_{e}\right. \\
& +\bar{e}\left(\left(-\frac{1}{2}+\frac{g_{0}^{2}}{2 g^{2}}\right)(1+2 x) \gamma^{\mu}-\frac{1}{2}\left(1-\frac{2\left(g^{2}+g_{0}^{2}\right)}{g^{2}} x\right) \gamma^{\mu} \gamma^{5}\right) e \\
& \left.+\mathcal{O}\left(x^{2}\right)+\mathcal{O}\left(g_{A}^{2}\right)\right] Z_{\mu}^{(2)},
\end{aligned}
$$

where $e_{0}=g g_{0} / \sqrt{2\left(g^{2}+g_{0}^{2}\right)}$ is the positron electric charge.

Any extension of some known chiral gauge model demands new consideration of a question on the axial anomaly of Adler-Bell-Jackiw [15, 16], which must be cancelled to avoid the breakdown of gauge invariance and renormalizability of the theory. The Adler-Bardeen theorem [17] guarantees that the axial anomaly only receives contribution from one loop diagrams, which are [18] the AVV or AAA triangle diagrams. Freedom from the triangular chiral gauge anomaly was first proved for the Weinberg-Salam model with $S U(2)_{L} \otimes U(1)[19,20]$, then 
for the standard model with $S U(3)_{c} \otimes S U(2)_{L} \otimes U(1)$ and the left-right symmetric model with $S U(3)_{c} \otimes S U(2)_{L} \otimes S U(2)_{R} \otimes U(1)$ (see, for example [21]). It is remarkable that in all mentioned cases, the anomaly exactly cancels between leptons and quarks for each their family. The anomaly-free conditions do not demand the existence of several generations of fundamental fermions.

In our initially $P$-invariant electroweak model with light (massless) axial gauge boson, the gauge group is locally isomorphic to the group $S U(2)_{L} \otimes S U(2)_{R} \otimes U(1) \otimes U(1)_{A}$. So, it is enough to consider the conditions of the axial anomaly cancellation for triangular diagrams with one, two, or three axial gauge bosons. It is easy to be convinced that such cancellation will necessarily occur, at least, when the constant $c_{2}$ from equations (7), (9), (10), and (11) is equal to zero, and the constant $c_{1}$ from equations (5), (6), and (11) is equal to zero for one of the three lepton (quark) generations and takes opposite values for the other two. Note that nonzero values of the constant $\left|c_{1}\right|$ for leptons and quarks are not connected in any way with each other.

\section{Existing models with a light or very light gauge boson}

A gauge boson, which may be very light and very weakly coupled, was proposed a long time ago in the context of a supersymmetric theory as the spin-1 partner of the goldstino (gravitino) $[22,23]$. It was supposed, that the new boson is axially coupled to leptons and quarks.

A new light gauge boson introduced also by the consideration of an extra $U(1)$ gauge symmetry for extension of the Weinberg-Salam model or the left-right symmetric model [24]. Let us notice that in the work [24] the extra gauge group is $U(1)$, but is not $U(1)_{A}$. Therefore this gauge boson interacts with the current, which is pure vector or it also includes an axial part.

Some experimental consequences of the possible existence of a new light gauge boson are discussed in the paper [25]. I would like to add the following to it.

The fundamental question, it would be desirable to receive an exhaustive answer to, concerns an old problem of the solar neutrino (see, for example, the review [26]). Can the interaction of the light (massless) axial gauge boson give, as a result, such energy spectrum of the solar neutrino which leads to the reduction of the observable rate of transitions ${ }^{37} \mathrm{Cl} \rightarrow{ }^{37} \mathrm{Ar}$ and ${ }^{71} \mathrm{Ga}$ $\rightarrow{ }^{71} \mathrm{Ge}$ in comparison with the rate expected in the standard solar model?

It is admissible to suppose that the light axial gauge boson contribution to the elastic scattering of reactor antineutrinos on electrons is comparable with the $Z$-boson contribution. This statement is based on that the charged current gives the dominating contribution in the cross section of $\bar{\nu}_{e} e$ elastic scattering [27], and the experimental errors and background are large enough $[28,29]$. If $c_{1}=2 \sqrt{2}$ for the electronic isodoublet, then the corresponding estimation of the $\bar{\nu}_{e} e$ coupling constant gives the following upper limit

$$
\alpha_{A} \equiv \frac{g_{A}^{2}}{4 \pi} \sim 10^{-8} \alpha
$$

where $\alpha$ is the fine-structure constant. It is necessary to emphasize especially that this estimation does not contradict to the experimental value for the gyromagnetic quantity $g-2$ of the electron [13], having in mind the replacement of the standard formula [30] $g-2=\alpha / \pi+\mathcal{O}\left(\alpha^{2}\right)$ with the formula $g-2=\alpha / \pi+\alpha_{A} / \pi+\mathcal{O}\left(\alpha^{2}\right)+\mathcal{O}\left(\alpha_{A}^{2}\right)$. For the letter expression, the value of $\alpha$ must be extracted from all available experimental data [31], except the ones regarding the $g-2$ factor itself.

\section{Acknowledgements}

I am very grateful to S.P. Baranov and N.P. Yudin for useful discussions on this report. 
[1] Slad L.M., Double symmetries in field theories, Mod. Phys. Lett. A, 2000, V.15, 379-389, hep-th/0003107.

[2] Gell-Mann M., Levy M., The axial vector current in beta decay, Nuovo Cimento, 1960, V.16, 705-726.

[3] Slad L.M., Toward an infinite-component field theory with a double symmetry: Free fields, Theor. Math. Phys., 2001, V.129, 1369-1384, hep-th/0111140.

[4] Slad L.M., Toward an infinite-component field theory with a double symmetry: Interaction of fields, Theor. Math. Phys., 2002, V.133, 1363-1375, hep-th/0210120.

[5] Slad L.M., Double symmetry and infinite-component field theory, in Proceedinds of Fifth International Conference "Symmetry in Nonlinear Mathematical Physics" (June 23-29, 2003, Kyiv), Editors A.G. Nikitin, V.M. Boyko, R.O. Popovych, and I.A. Yehorchenko, Proceedings of Institute of Mathematics, Kyiv, 2004, V.50, Part 2, 947-954, hep-th/0312273.

[6] Slad L.M., Mass spectra in the doubly symmetric theory of infinite-component fields, Theor. Math. Phys., 2005, V.142, 15-28, hep-th/0312150.

[7] Ginzburg V.L., On relativistic wave equations with mass spectrum, Acta Physica Polonica, 1956, V.15, 163-175.

[8] Gelfand I.M., Minlos R.A., Shapiro Z.Ya., Representations of the rotation and Lorenz group and their applications, New York, The Macmillan Company, 1963.

[9] Pati J.C., Salam A., Lepton number as the fourth "color", Phys. Rev. D, 1974, V.10, 275-289.

[10] Mohapatra R.N., Pati J.C., Left-right gauge symmetry and an "isoconjugate" model of $C P$ violation, Phys. Rev. D, 1975, V.11, 566-571.

[11] Mohapatra R.N., Pati J.C., "Natural" left-right symmetry, Phys. Rev. D, 1975, V.11, 2558-2561.

[12] Senjanovic G., Mohapatra R.N., Exact left-right symmetry and spontaneous violation of parity, Phys. Rev. D, 1975, V.12, 1502-1505.

[13] Eidelman S. et al., Review of particle physics, Phys. Lett. B, 2004, V.592, 1-1109.

[14] Weinberg S., A model of leptons, Phys. Rev. Lett., 1967, V.19, 1264-1266.

[15] Adler S.L., Axial-vector vertex in spinor electrodynamics, Phys. Rev., 1969, V.177, 2426-2438.

[16] Bell J., Jackiw R., A PCAC puzzle: $\pi^{0} \rightarrow \gamma \gamma$ in the sigma model, Nuovo Cimento A, 1969, V.60, 47-61.

[17] Adler S.L., Bardeen W.A., Absence of higher-order corrections in the anomalous axial-vector divergence equation, Phys. Rev., 1969, V.182, 1517-1536.

[18] Adler S.L., Anomalies, hep-th/0411038.

[19] Gross D.J., Jackiw R., Effect of anomalies on quasi-renormalizable theories, Phys. Rev. D, 1972, V.6, 477493.

[20] Bouchiat C., Iliopoulos J., Meyer Ph., An anomaly-free version of Weinberg's model, Phys. Lett. B, 1972, V.38, 519-523.

[21] Geng C.Q., Marshak R.E., Uniqueness of quark and lepton representations in the standard model from the anomalies viewpoint, Phys. Rev. D, 1989, V.39, 693-696.

[22] Fayet P., Effects of the spin-1 partner of the goldstino (gravitino) on neutral current phenomenology, Phys. Lett. B, 1980, V.95, 285-289.

[23] Fayet P., A la recherche d'un nouveau boson de spin un, Nucl. Phys. B, 1981, V.187, 184-204.

[24] Fayet P., Extra U(1)'s and new forces, Nucl. Phys. B, 1990, V.347, 743-768.

[25] Boehm C., Implications of a new light gauge boson for neutrino physics, Phys. Rev. D, 2004, V.70, 055007, 9 pages.

[26] Bellerive A., Review of solar neutrino experiments, Int. J. Mod. Phys. A, 2004, V.19, 1167-1179.

[27] Marciano W.J., Parsa Z., Neutrino-electron scattering theory, J. Phys. G, 2003, V.29, 2629-2646.

[28] Reines F., Curr H.S., Sobel H.W., Detection of $\bar{\nu}_{e}-e$ scattering, Phys. Rev. Lett., 1976, V.37, 315-318.

[29] Daraktchieva Z. et al., Final results on the neutrino magnetic moment from the MUNU experiment, Phys. Lett. B, 2005, V.615, 153-159.

[30] Levine M.J., Park H.Y., Roskies R.Z., High-precision evaluation of contributions to $g-2$ of the electron in sixth order, Phys. Rev. D, 1982, V.25, 2205-2207.

[31] Mohr P.J., Taylor B.N., CODATA recommended values of the fundamental physical constants: 2002, Rev. Mod. Phys., 2005, V.77, 1-107. 